\title{
Universal Rashba spin precession of two-dimensional electrons and holes
}

\author{
Marco G. Pala ${ }^{1,2}$, Michele Governale ${ }^{2}$, Jürgen König ${ }^{2}$ and Ulrich Zülicke ${ }^{2}$ \\ 1 Dipartimento di Ingegneria dell'Informazione, via Diotisalvi 2, I-56126 Pisa, Italy \\ 2 Institut für Theoretische Festkörperphysik, Universität Karlsruhe, D-76128 Karlsruhe, \\ Germany
}

PACS. 85.75. Hh - Spin polarized field effect transistors.

PACS. 72.25.-b - Spin polarized transport.

PACS. 73.23. Ad - Ballistic transport.

\begin{abstract}
We study spin precession due to Rashba spin splitting of electrons and holes in semiconductor quantum wells. Based on a simple analytical expression that we derive for the current modulation in a broad class of experimental situations of ferromagnet/nonmagnetic semiconductor/ferromagnet hybrid structures, we conclude that the Datta-Das spin transistor (i) is feasible with holes and (ii) its functionality is not affected by integration over injection angles. The current modulation shows a universal oscillation period, irrespective of the different forms of the Rashba Hamiltonian for electrons and holes. The analytic formulas approximate extremely well exact numerical calculations of a more elaborate Kohn-Luttinger model.
\end{abstract}

Transport effects based on coherent manipulation of the spin degree of freedom in lowdimensional semiconductors are currently attracting a lot of attention [1]. These studies, enabled by recent progress in nanofabrication technology to create high-quality samples, are motivated by both their interesting fundamental physics and their potential for future device applications [2]. A lot of progress in the field has been stimulated by the exploitation of spin precession due to Rashba spin-orbit (SO) coupling in 2D systems both for electrons [3-5] and for holes [6]. A prominent example is the spin-controlled field-effect transistor (spin FET) introduced by Datta and Das [7], followed by more recent proposals for novel devices utilizing Rashba SO coupling [8]. Both in the original [7] and most subsequent [9-14] works, a quasionedimensional (1D) confinement was considered essential for proper spin-FET action. Spin precession in truly 2D electron systems was studied numerically in a number of works $[15,16]$. On the other hand, ever-present spin relaxation will reduce the spin polarization of currents, preventing the realization of gate-controlled modulation. Such processes arise, e.g., from magnetic impurities but most importantly from elastic impurity scattering that randomizes the direction of the effective Rashba field. Stronger spin-orbit coupling and band mixing phenomena imply a shorter spin relaxation time for the holes respect to the electrons, that can be compensated by shorter precession length. A nice proposal, which exploits tunability of Rashba SO coupling, to overcome the detrimental effects due to scattering processes is presented in the last paper of Ref. [8].

(C) EDP Sciences 


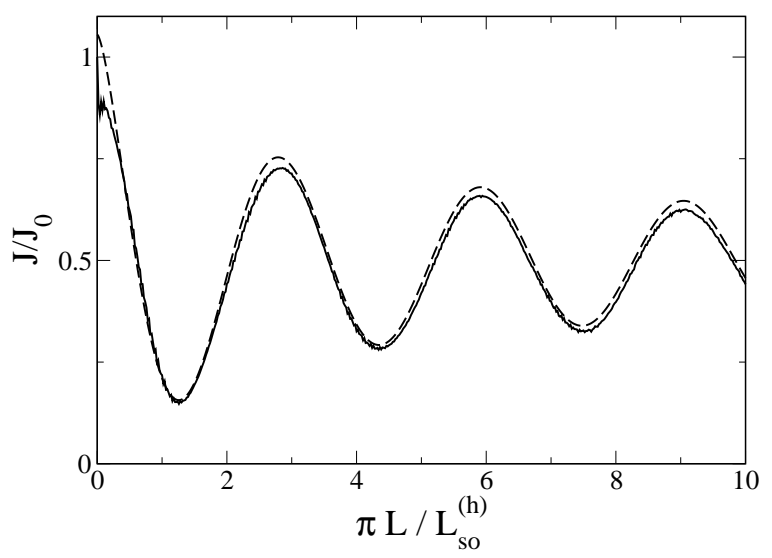

Fig. 1 - Current modulation in a p-type 2D ferromagnet/nonmagnetic-semiconductor/ferromagnet hybrid system computed numerically (solid line) for a realistic system and compared with our analytic expression (dashed line). Here $L$ is the width of the nonmagnetic region, and $L_{\mathrm{so}}^{(\mathrm{h})}$ the spin-precession length for holes due to the Rashba effect. The magnetizations of the two ferromagnetic regions are parallel and point in a direction perpendicular to the 2D plane of the hybrid system. For the numerical simulation we have used parameter values for a realistic GaMnAs/GaAs/GaMnAs 2D heterostructure. The hole densities are of order $10^{16} \mathrm{~m}^{-2}, L_{\mathrm{so}}^{(\mathrm{h})} \approx 300 \mathrm{~nm}$, and the exchange field is $150 \mathrm{meV}$. There is a Fermi wave vector mismatch $k_{\mathrm{F}}^{(\mathrm{f})} / k_{\mathrm{F}, 0}=1.33$; and the Fermi wave vector splitting due to SO coupling is $\Delta k / k_{\mathrm{F}, 0}=7.66 \cdot 10^{-3}$, corresponding to $\beta_{\mathrm{p}}\left\langle E_{z}\right\rangle=1.3 \cdot 10^{20} \mathrm{eV}^{-1} \mathrm{sec}^{-2} \mathrm{~nm}$.

The aim of this Letter is twofold. First, we provide a unified analytical description of spin precession for both electrons and holes in the case of ballistic transport regime, discussing common universal features and retaining the $2 \mathrm{D}$ nature of the problem. This is the most realistic case for anticipated device applications. One of our main results is illustrated in Fig. 1 where our approximate analytical expression for the current modulation in a 2D hole spin FET is compared with the full numerical result obtained by mode-matching within a more elaborate Kohn-Luttinger model. Except for very small values of the distance $L$ between the ferromagnetic contacts, the agreement is excellent. We prove analytically that the oscillation period in the $2 \mathrm{D}$ setup is the same as in the quasi-1D structures considered before $[7,9]$. Second, we want to emphasize the utility of holes as carriers in a spin FET. The apparent disadvantage of a shorter spin life time as compared to electrons is off-set by shorter spin-precession lengths and, above all, by the possibility to use recently created [17] p-type ferromagnetic semiconductors as current injectors. Besides obvious advantages concerning integrability in current semiconductor technology, such an all-semiconductor spin FET would circumvent the problems [18] that prevent spin injection from metallic magnets into semiconductors in the absence of a large interface barrier.

We now turn to explaining details of our calculations. A 2 D hybrid system with two semiinfinite ferromagnetic contacts separated by a nonmagnetic $2 \mathrm{D}$ stripe of width $L$ is considered. To be specific, the extension of both ferromagnetic and nonmagnetic parts is infinite in $y$ direction, and the nonmagnetic stripe is bounded by interfaces to the ferromagnetic contacts at $x=0$ and $x=L$. Due to structural inversion asymmetry in the growth $(z)$ direction, charge carriers are subject to a spin-orbit coupling of the Rashba type $[3,5,6]$. For electrons in the s-like conduction band, it reads $\beta_{\mathrm{s}}\left\langle E_{z}\right\rangle(\vec{p} \times \vec{\sigma}) \cdot \hat{z}$, while for the $\mathrm{p}$-like valence bands we have $\beta_{\mathrm{p}}\left\langle E_{z}\right\rangle(\vec{p} \times \vec{J}) \cdot \hat{z}[19,20]$. Here, $\left\langle E_{z}\right\rangle$ is related to the average of the electric field in growth 

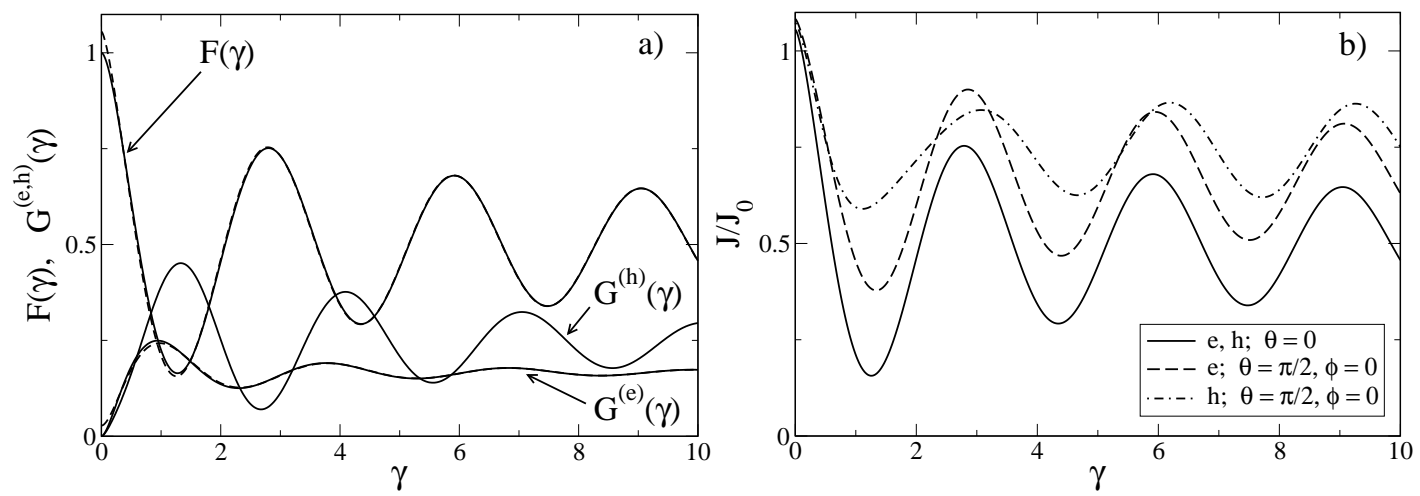

Fig. 2 - a) Plot of the functions $F(\gamma)$ and $G^{(\mathrm{e}, \mathrm{h})}(\gamma)$ computed numerically (solid lines) and by means of the approximated formulae given in the text (dashed line). The difference between analytical and numerical results is only barely visible for $\gamma \rightarrow 0$. b) Current density as a function of $\gamma=\pi L / L_{\mathrm{so}}^{(\mathrm{e}, \mathrm{h})}$ for different magnetization directions for both the electron and hole cases. The current is computed using the approximated expressions for $F(\gamma)$ and $G^{(\mathrm{e}, \mathrm{h})}(\gamma)$.

direction. In 2D hole quantum wells, the degeneracy of heavy-hole (HH) and light-hole (LH) valence bands at the zone center is lifted by the vertical confinement [21], and only the first $\mathrm{HH}$ subband $\left(\mathrm{HH}_{1}\right)$ is populated for typical $2 \mathrm{D}$ sheet densities. In the following, we consider only the $\mathrm{HH}_{1}$ subband unless specified otherwise. The effective Hamiltonians for Rashba SO coupling experienced by electrons and holes are $[19,20]$

$$
\begin{aligned}
& H_{\mathrm{so}}^{(\mathrm{e})}=\beta_{\mathrm{s}}\left\langle E_{z}\right\rangle i\left[p_{-} \sigma_{+}-p_{+} \sigma_{-}\right] \\
& H_{\mathrm{so}}^{(\mathrm{h})}=\beta_{\mathrm{h}}\left\langle E_{z}\right\rangle i\left[p_{-}^{3} \sigma_{+}-p_{+}^{3} \sigma_{-}\right],
\end{aligned}
$$

where $p_{ \pm}=p_{x} \pm i p_{y}$ are linear combinations of momentum components, $\sigma_{ \pm}=\left(\sigma_{x} \pm i \sigma_{y}\right) / 2$ denote Pauli matrices, and $\beta_{\mathrm{s}, \mathrm{h}}$ are material parameters.

We assume identical ferromagnetic contacts having parallel magnetization $M$ whose direction (in 3D) is described by two polar angles $(\theta, \phi)$, where $\phi=0$ corresponds to magnetization perpendicular to interface. As quasi-2D ferromagnets have either easy-axis or easy-plane anisotropy, the two relevant cases are that with magnetization perpendicular to the $2 \mathrm{D}$ plane $(\theta=0)$ or in the plane $(\theta=\pi / 2)$. Assuming that there is no reflection at the interfaces between the nonmagnetic semiconductor and the ferromagnetic contacts, we can calculate the transmission probability $T_{\theta, \phi}(\alpha)$ for an electron impinging on the first interface at an angle $\alpha$. (Details and a discussion of the approximation are given further below.) The total current density is then $J \propto \int_{-\pi / 2}^{\pi / 2} T_{\theta, \phi}(\alpha) \cos (\alpha) d \alpha$. We find its expression for arbitrary $(\theta, \phi)$ as

$$
J=J_{0}\left\{\cos ^{2} \theta F(\gamma)+\sin ^{2} \theta\left[\sin ^{2} \phi+F(\gamma) \cos ^{2} \phi+G^{(\mathrm{e}, \mathrm{h})}(\gamma) \cos (2 \phi)\right]\right\},
$$

where the functions $F$ and $G^{(\mathrm{e}, \mathrm{h})}$ are shown in the panel a) of Fig. 2] and are defined explicitly below. Here $\gamma=\pi L / L_{\mathrm{so}}^{(\mathrm{e}, \mathrm{h})}$, where $L_{\mathrm{so}}^{(\mathrm{e}, \mathrm{h})}=2 \pi / \Delta k$ is the spin precession length for electrons or holes, with $\Delta k$ being the difference of Fermi wave vectors for the spin-split 2D subbands:

$$
\begin{aligned}
L_{\mathrm{so}}^{(\mathrm{e})} & =\frac{\pi}{\beta_{\mathrm{s}}\left\langle E_{z}\right\rangle}\left(\frac{\hbar}{m^{(\mathrm{e})}}\right), \\
L_{\mathrm{so}}^{(\mathrm{h})} & =\frac{\pi}{2 \beta_{\mathrm{h}}\left\langle E_{z}\right\rangle}\left(\frac{\hbar}{m^{(\mathrm{h})}}\right)^{2} \frac{1}{\epsilon_{\mathrm{F}}} .
\end{aligned}
$$


Here $m^{(\mathrm{e}, \mathrm{h})}$ is the effective mass for electrons/holes, and $\epsilon_{\mathrm{F}}$ is the Fermi energy. Equation (3b) is valid when the Rashba spin splitting is small compared to the Fermi energy. Equation (2) is one of the central results of this Letter, and a few remarks on it are in order. Once we decide whether the carriers are electrons or holes and, hence, fix the symmetry of the band ( $\mathrm{s}$ or $\mathrm{p}$ type), the current density has a universal behavior: it depends only on the ratio of the distance between the ferromagnetic contacts and the spin-precession length. Finite transparency of the interfaces and Fermi-velocity mismatch between the ferromagnetic and nonmagnetic materials will be shown below to lead only to small quantitative changes. The only difference in the functional form of the current for electrons and holes is in $G^{(\mathrm{e}, \mathrm{h})}(\gamma)$ and thus appears only if the magnetization has an in-plane component. The current density Eq. (2) shows oscillations in $\gamma$ with a period $\pi$, i.e., oscillations in $L$ with a period $L_{\mathrm{so}}^{(\mathrm{e}, \mathrm{h})}$, that are a manifestation of spin precession in the nonmagnetic region. A few observations can be made regarding the behavior of the oscillatory part of the current density for different magnetization directions (see panel b) of Fig. 21): i) The largest oscillations of the current (solid line in the plot) as a function of $\gamma$ occur for magnetization perpendicular to the 2D system $(\theta=0)$; ii) In this case electrons and holes behave exactly in the same way; iii) For in-plane magnetization perpendicular to the interface $(\theta=\pi / 2, \phi=0)$, the electron system (dashed line in the plot) exhibits a slightly larger modulation of the conductance as compared to the hole system (dash-dotted line in the plot); iv) For in-plane magnetization parallel to the interface $(\theta=\pi / 2, \phi=\pi / 2)$ the conductance still shows oscillations as a function of $\gamma$ (this is in contrast to the case of normal incidence), which are larger for holes than electrons. The different functional form for the SO term for electron and holes [see Eq.(1)] does not affect the transmission probability for normal injection. Hence, the non universal features, when present, are due to the integration over the injection angle $\alpha$. We can conclude that the oscillations of the conductance as a function of $L_{\mathrm{so}}^{(\mathrm{e})}$ h) $a r e$ not washed out by the $2 \mathrm{D}$ geometry of the system, i.e., by the necessity to integrate over the direction of the incoming electrons in the ferromagnet. This oscillatory behavior of the current is exploited by the spin FET. Hence, our results show analytically that in the case of ballistic transport regime the spin FET does not require a quasi-1D setup to work properly, and that the largest modulations of the conductance as a function of the spin precession length are obtained for magnetization perpendicular to the 2D system. Furthermore, Eq. (2) proves the feasibility of a p-type spin-FET, with holes performing even better than electrons for certain magnetization directions.

To illustrate the strategy employed to obtain the general formula Eq. (2) without inflicting lengthy algebraic manipulations to the reader, it is sufficient to show the calculation for the case of a hole system and magnetization perpendicular to the plane $(\theta=0)$. We start by calculating the probability for a plane wave in the left ferromagnet, impinging on the first interface at an angle $\alpha$, to be transmitted in the second ferromagnet. We assume that only majority spins contribute to transport, hence the spin state of the incoming electron in the left ferromagnet is $|+\rangle$, where $|+\rangle$ is the spinor corresponding to spin in the $+z$ direction (the magnetization direction). We now need to write the wave function in the semiconductor. We notice first that the SO coupling Hamiltonian Eq. (1b) removes the degeneracy of $\mathrm{HH}_{1}$ subbands. The eigenstates are still plane waves $\exp [i k(x \cos \alpha+y \sin \alpha)] \chi_{ \pm}$, with spinors $\chi_{ \pm}=(1 / \sqrt{2})[|+\rangle \mp i \exp (i 3 \alpha)|-\rangle]$. The spin-split dispersion relations associated to these states read $\epsilon_{ \pm}=\frac{\hbar^{2}}{2 m^{(\mathrm{h})}} k^{2} \pm \beta_{\mathrm{h}}\left\langle E_{z}\right\rangle \hbar^{3} k^{3}$. As we study linear transport, we are interested in the states at the Fermi energy. In particular, the Fermi wave vectors for the $\epsilon_{ \pm}$bands are $k_{\mathrm{F}, \pm}=k_{\mathrm{F}, 0} \mp \Delta k / 2$ where $k_{\mathrm{F}, 0}$ is the Fermi wave vector when no SO coupling is present. Due to translational invariance along the interface, the wave vector component parallel to the interface is conserved when going from the ferromagnet to the non magnetic semiconductor. A plane 
wave in the ferromagnet with $\vec{k}=k_{\mathrm{F}}^{(\mathrm{f})}(\hat{x} \cos \alpha+\hat{y} \sin \alpha)$ gives rise to two transmitted waves with propagation directions defined by angles $\alpha_{ \pm}$. This effect is similar to birefringence [10, 22]: the bands $\epsilon_{ \pm}$have different Fermi wave vectors, resulting in two different propagating directions for the transmitted waves. In the limit of weak SO coupling, i.e., $\Delta k / k_{\mathrm{F}, 0} \ll 1$, the two angles read $\alpha_{ \pm}=\alpha_{0} \pm(1 / 2)\left(\Delta k / k_{\mathrm{F}, 0}\right) \tan \alpha_{0}$, and $\alpha_{0}$ is defined by $k_{\mathrm{F}}^{(\mathrm{f})} \sin \alpha=$ $k_{\mathrm{F}, 0} \sin \alpha_{0}$. We are now in the position to write the transmitted state in the nonmagnetic strip: it simply reads $c_{+} \exp \left[i k_{\mathrm{F},+}\left(x \cos \alpha_{+}+y \sin \alpha_{+}\right)\right] \chi_{+}+c_{-} \exp \left[i k_{\mathrm{F},-}\left(x \cos \alpha_{-}+y \sin \alpha_{-}\right)\right] \chi_{-}$. By assuming perfect transparency of the interface we can compute the coefficients $c_{ \pm}$simply by matching the wave functions in the ferromagnet and non magnetic semiconductor. At the other interface $x=L$, only the $|+\rangle$ component will be transmitted, hence the outgoing state in the right ferromagnet reads $\exp \left[i k_{\mathrm{F}}^{(\mathrm{f})}(x \cos \alpha+y \sin \alpha)\right] \cos \left[\Delta k L /\left(2 \cos \alpha_{0}\right)\right]|+\rangle$. From that the transmission probability can be read off:

$$
T_{0, \phi}(\alpha)=\cos ^{2}\left[\frac{\gamma}{\cos \alpha_{0}}\right]
$$

where we have used the relation $\Delta k L / 2=\gamma$, and the dependence on $\alpha$ is through $\alpha_{0}$ via the relation $k_{\mathrm{F}}^{(\mathrm{f})} \sin \alpha=k_{\mathrm{F}, 0} \sin \alpha_{0}$. In a similar way we can obtain the transmission probabilities for the electron case and for arbitrary magnetization direction. We find that Eq. (4) is valid for both electrons and holes. The transmission probability for in-plane magnetization reads

$$
T_{\pi / 2, \phi}(\alpha)=\cos ^{2}\left[\frac{\gamma}{\cos \alpha_{0}}\right]+\sin ^{2}\left[\nu_{\mathrm{e}, \mathrm{h}} \alpha_{0}-\phi\right] \sin ^{2}\left[\frac{\gamma}{\cos \alpha_{0}}\right] \text {, }
$$

where $\nu_{\mathrm{e}}=1$ and $\nu_{\mathrm{h}}=3$. Finally, we can write the transmission for arbitrary magnetization direction as

$$
T_{\theta, \phi}(\alpha)=\cos ^{2} \theta T_{0, \phi}+\sin ^{2} \theta T_{\pi / 2, \phi}
$$

Equations (4 6) cease to be valid once one of the transmitted states in the nonmagnetic strip becomes evanescent and is totally reflected. This condition defines the critical angles $\alpha_{\mathrm{c}, \pm}$, that in the limit of weak SO coupling read $\alpha_{\mathrm{c}, \pm}=\frac{k_{\mathrm{F}, 0}}{k_{\mathrm{F}}^{(\mathrm{f})}} \mp \frac{1}{2} \frac{\Delta k}{k_{\mathrm{F}}^{(\mathrm{f})}} \approx \frac{k_{\mathrm{F}, 0}}{k_{\mathrm{F}}^{(\mathrm{f})}}=\alpha_{\mathrm{c}}$. At this point a few remarks on the approximate analytical treatment are in order: i) In this calculation scheme the spin precession in the nonmagnetic strip and the spin selecting properties of the ferromagnets (they act as polarizer and analyzer) are fully taken into account, while the interference effects arising from multiple reflection between the interfaces are neglected. This is in the same spirit of the calculation of Datta and Das [7] and of all the quantum-mechanical Gedanken experiments involving polarized photons and polarizers. Indeed, multiple reflection introduces a modulation of the transmission coefficients $\propto \cos ^{2}\left(L k_{\mathrm{F}} / \cos \alpha\right)$. ii) This fast oscillation is washed out by the integration over the injection angle. This explains the remarkable agreement with the full quantum-mechanical calculation, shown in Fig. 1]

The current density perpendicular to the interface is proportional to $\int_{-\alpha_{\mathrm{c}}}^{\alpha_{\mathrm{c}}} T_{\theta, \phi}(\alpha) \cos \alpha d \alpha \propto$ $\int_{-\pi / 2}^{\pi / 2} \tilde{T}_{\theta, \phi}\left(\alpha_{0}\right) \cos \alpha_{0} d \alpha_{0}$, where $\tilde{T}_{\theta, \phi}\left(\alpha_{0}\right)=T_{\theta, \phi}\left(\alpha\left(\alpha_{0}\right)\right)$. Writing this integral with $T_{\theta, \phi}(\alpha)$ given in Eqs. (4 6), we obtain Eq. (2), where $F(\gamma)$ and $G^{(\mathrm{e}, \mathrm{h})}$ are

$$
\begin{gathered}
F(\gamma)=\frac{1}{2} \int_{-\frac{\pi}{2}}^{\frac{\pi}{2}} \cos \alpha \cos ^{2}\left(\frac{\gamma}{\cos \alpha}\right) d \alpha \\
G^{(\mathrm{e}, \mathrm{h})}(\gamma)=\frac{1}{2} \int_{-\frac{\pi}{2}}^{\frac{\pi}{2}} \cos \alpha \sin ^{2}\left(\nu_{\mathrm{e}, \mathrm{h}} \alpha\right) \sin ^{2}\left(\frac{\gamma}{\cos \alpha}\right) d \alpha
\end{gathered}
$$


We have obtained an approximate analytical solution of Eqs. (7) and (8) that is valid unless $\gamma$ is very small:

$$
\begin{aligned}
F(\gamma)= & \frac{\pi \sqrt{2}}{8}\left\{(4 \gamma-1) \mathrm{S}\left(2 \sqrt{\frac{\gamma}{\pi}}\right)+(-4 \gamma+1) \mathrm{C}\left(2 \sqrt{\frac{\gamma}{\pi}}\right)+\right. \\
& \left.2 \sqrt{\frac{\gamma}{\pi}}[\cos (2 \gamma)+\sin (2 \gamma)]+1+\frac{4}{\pi \sqrt{2}}\right\}, \\
G^{(\mathrm{e}, \mathrm{h})}(\gamma)= & \sqrt{2} \pi\left\{P^{(\mathrm{e}, \mathrm{h})}(\gamma) \mathrm{C}\left(2 \sqrt{\frac{\gamma}{\pi}}\right)+P^{(\mathrm{e}, \mathrm{h})}(-\gamma) \mathrm{S}\left(2 \sqrt{\frac{\gamma}{\pi}}\right)-\right. \\
& \left.\sqrt{\frac{\gamma}{\pi}}\left[Q^{(\mathrm{e}, \mathrm{h})}(\gamma) \cos (2 \gamma)+Q^{(\mathrm{e}, \mathrm{h})}(-\gamma) \sin (2 \gamma)\right]+M^{(\mathrm{e}, \mathrm{h})}(\gamma)\right\},
\end{aligned}
$$

where $C$ and $S$ are cosine and sine Fresnel integrals [23], respectively, and

$$
\begin{aligned}
& P^{(\mathrm{e})}(\gamma)=\frac{2}{3} \gamma^{3}-\frac{1}{2} \gamma^{2}+\frac{1}{8} \gamma+\frac{1}{32}, \\
& P^{(\mathrm{h})}(\gamma)=\frac{61}{315} \gamma^{7}-\frac{16}{45} \gamma^{6}+\frac{4}{3} \gamma^{5}-\gamma^{4}+\frac{11}{12} \gamma^{3}-\frac{7}{16} \gamma^{2}+\frac{9}{64} \gamma+\frac{13}{256}, \\
& Q^{(\mathrm{e})}=\frac{1}{3} \gamma^{2}+\frac{1}{6} \gamma+\frac{1}{16}, \\
& Q^{(\mathrm{h})}=\frac{32}{315} \gamma^{6}+\frac{16}{105} \gamma^{5}+\frac{218}{315} \gamma^{4}+\frac{34}{105} \gamma^{3}+\frac{11}{24} \gamma^{2}+\frac{7}{48} \gamma+\frac{13}{128}, \\
& M^{(\mathrm{e})}(\gamma)=\frac{1}{2} \gamma^{2}-\frac{1}{32}+\frac{1}{6 \pi \sqrt{2}}, \\
& M^{(\mathrm{h})}(\gamma)=\frac{16}{45} \gamma^{6}+\gamma^{4}+\frac{7}{16} \gamma^{2}+\frac{17 \sqrt{2}}{140 \pi}-\frac{13}{256} .
\end{aligned}
$$

In Fig. 2] we compare the approximate results Eqs. (9) and (10) with the integrals of Eqs. (77) and (8). The approximate formulae work extremely well: the largest errors are for $\gamma=0$, and hence not in the region of physical interest, and the errors decrease monotonically with increasing $\gamma$. The relative errors for $\gamma=\pi$, i.e., after a full precession, are of order $10^{-3}$ [24].

We have derived the above results for transmission probabilities [Eqs. [6]] and the current density [Eq. (2)] making use of several approximations, such as perfectly transparent interfaces and, in the hole case, the truncation to the lowest heavy-hole subband. We now test the accuracy of our approximate results by comparing them to a more realistic model for the hole system which is the more complicated one. Both the magnetic and nonmagnetic parts of the hybrid system are modeled by a $4 \times 4$ Kohn-Luttinger Hamiltonian with $\mathrm{HH}$ and LH bands. The confinement in the growth direction is treated by means of the envelopefunction approximation. The ferromagnetic contacts are described within the Stoner approach by an exchange field. Calculation of transmission and reflection coefficients by means of a full quantum-mechanical mode matching at the two interfaces and integration over angle of incidence yields the current density shown in Fig. [1 as a solid line. Excellent agreement between our approximate analytical results and the exact numerical curve is apparent (note that for the latter a Fermi wave vector mismatch across the interface is present.) The only effect of nonideal interfaces on the current modulation is a renormalization of its amplitude $J_{0}$.

In conclusions, we have studied spin precession in electron and hole system, finding a universal expression for the current in a spin FET geometry. The full 2D nature of the 
problem is retained. Our results show that the current through the system is determined only by the common magnetization direction in the ferromagnets and by the ratio of the distance between the ferromagnetic contacts and the spin-precession length.

This work was supported by DFG through the Center for Functional Nanostructures. M. P. acknowledges support from the IST NanoTCAD project (EC contract IST-1999-10828). We thank P. Mastrolia for suggesting a method to evaluate the integrals in Eqs. (7) and (8).

\section{REFERENCES}

[1] Awschalom D. D., Loss D., and Samarth N., Semiconductor Spintronics and Quantum Computation (Springer, Berlin) 2002.

[2] Wolf S. A. et al., Science, 294 (2001) 1488.

[3] Rashba E. I., Fiz. Tverd. Tela (Leningrad), 2 (1960) 1224; Sov. Phys. Solid State, 2 (1960) 1109.

[4] Bychkov Y. A. and Rashba E. I., J. Phys. C, 17 (1984) 6039.

[5] Lommer G., Malcher F. and Rössler U., Phys. Rev. Lett., 60 (1988) 728.

[6] Gerchikov L. G. and Subashiev A. V., Sov. Phys. Semicond., 26 (1992) 73.

[7] Datta S. and Das B., Appl. Phys. Lett., 56 (1990) 665.

[8] De Andrada e Silva E. A. and La Rocca G. C., Phys. Rev. B, 59 (1999) R15583; Nitta J., Meijer F. E. and Takayanagi H., Appl. Phys. Lett., 75 (1999) 695; Kiselev A. A. and Kim K. W., Appl. Phys. Lett., 78 (2001) 775; Koga T. et al., Phys.Rev. Lett., 88 (2002) 126601; Governale M. et al., Phys.Rev. B, 65 (2002) 140403(R); Egues J. C., Burkard G. and Loss D., Phys. Rev. Lett., 89 (2002) 176401; Schliemann J., Egues J. C. and Loss D., Phys. Rev. Lett., 90 (2003) 146801.

[9] Mireles F. and Kirczenow G., Phys. Rev. B, 64 (2001) 024426.

[10] Matsuyama T. et al., Phys. Rev. B, 65 (2002) 155322.

[11] Governale M. and Zülicke U., Phys. Rev. B, 66 (2002) 073311.

[12] Egues J. C., Burkard G. and Loss D., Appl. Phys. Lett., 82 (2003) 2658

[13] Häusler W., Phys. Rev. B, 63 (2001) 121310(R).

[14] Pareek T. P., Phys. Rev. B, 66 (2002) 193301.

[15] Bournel A., Dolfus P., Bruno P. and Hesto P., Eur. Phys. J. AP, 4 (1998) 1; Pareek T. P. and Bruno P., Phys. Rev. B, 65 (2002) 241305.

[16] Raichev O. E. and Debray P., Phys. Rev. B, 65 (2002) 085319

[17] Ohno H., Science, 281 (1998) 951.

[18] Schmidt G. et al., Phys. Rev. B, 62 (2000) R4790.

[19] Winkler R., Phys. Rev. B, 62 (2000) 4245.

[20] Winkler R. et al., Phys. Rev. B, 65 (2002) 155303.

[21] Chow W. W., Koch S. W. and Sargent III M., Semiconductor Laser Physics (SpringerVerlag, Berlin) 1997, p. 179211.

[22] Marigliano Ramaglia V. et al., cond-mat/0203569 Preprint, 2002.

[23] Abramowitz M. and Stegun I. A., Handbook of Mathematical Functions (Dover Publications, New York) 1974.

[24] The absolute errors at $\gamma=0$ for $F(\gamma), G^{(\mathrm{e})}(\gamma)$, and $G^{(\mathrm{h})}(\gamma)$ are, respectively, $\pi /(4 \sqrt{2})-1 / 2 \approx$ $5.54 \cdot 10^{-2}, 1 / 6-\pi /(16 \sqrt{2}) \approx 2.78 \cdot 10^{-2}$, and $13 / 70-13 \pi /(128 \sqrt{2}) \approx 1.72 \cdot 10^{-2}$. For $\gamma=\pi$, the relative errors for $F(\gamma), G^{(\mathrm{e})}(\gamma)$, and $G^{(\mathrm{h})}(\gamma)$ are, respectively, $1.83 \cdot 10^{-3}, 7.49 \cdot 10^{-3}$, and $7.19 \cdot 10^{-4}$. 